\title{
La emisora escolar: estrategia didáctica para desarrollar competencias comunicativas de lectura y escritura en estudiantes de básica primaria
}

\author{
The School Radio Station: Didactic Strategy to \\ Develop Communicative Reading and Writing \\ Competencies in Elementary Basic Students
}

Helman Yesid Nieto Duarte*

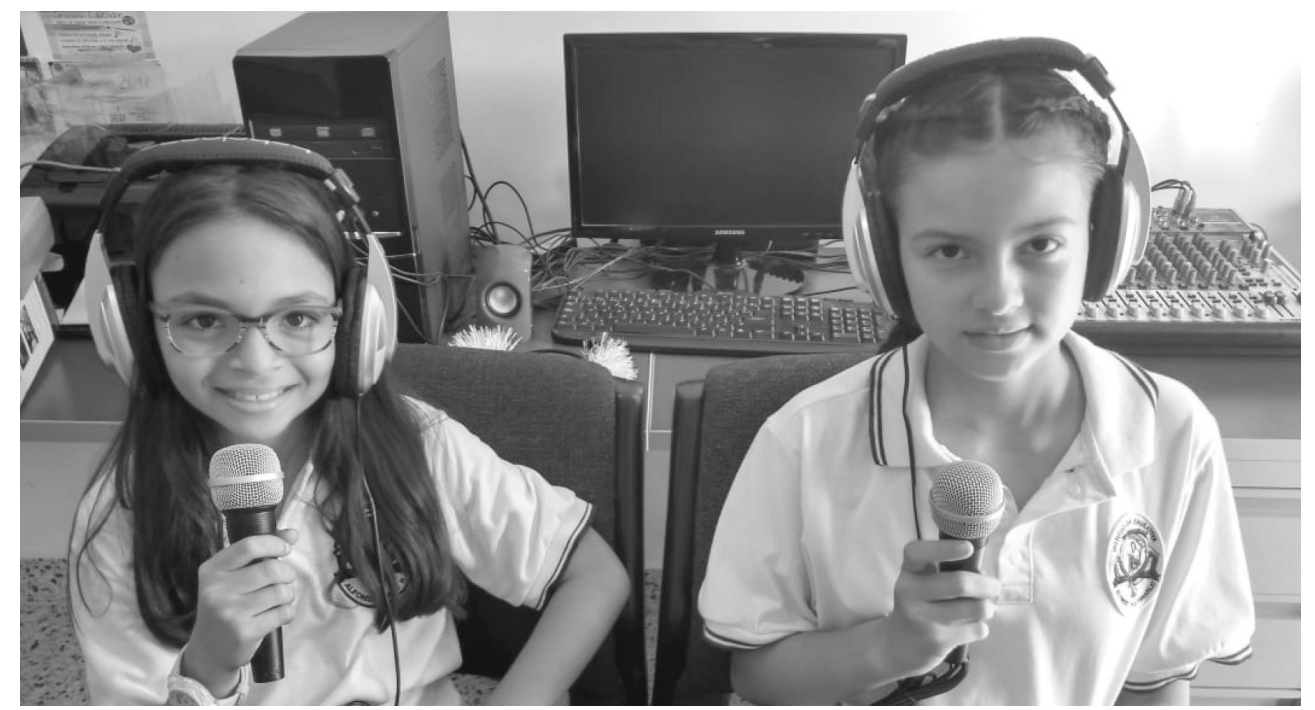

\section{Resumen}

Esta investigación, desarrollada en la Maestría en Educación de la Universidad Antonio Nariño (UAN) en Bogotá (Colombia), buscó desarrollar una estrategia que mediara en el desarrollo de las competencias comunicativas de lectura y escritura en las estudiantes del Liceo Femenino Mercedes Nariño IED (LFMN-IED), institución oficial de la ciudad. La investigación procuró exponer el proceso que desarrollan las

Citar este artículo como: Nieto Duarte, H. Y. (2020). La emisora escolar: estrategia didáctica para desarrollar competencias comunicativas de lectura y escritura en estudiantes de básica primaria Revista Papeles, 12(23), 98-111.

Fecha de recibido: 10 de marzo del 2020 Fecha de aprobado: mayo 15 de 2020

* Licenciado en Educación Básica con Énfasis en Lengua Castellana (Universidad del Tolima), Magíster en Educación (Universidad Antonio Nariño). Cuenta con experiencia de doce años en la práctica docente y la coordinación de instituciones educativas privadas y oficiales, en los niveles de educación básica y media, además de atención a población de jóvenes y adultos en sistema de educación flexible. Correo electrónico: hnieto16@uan.edu.co 
estudiantes con las habilidades comunicativas de la lectura y la escritura (HCLE) en las dinámicas propias de la emisora escolar, además de establecer por medio de un instrumento evaluativo las habilidades iniciales y las alcanzadas en las competencias mencionadas en los niveles semántico, sintáctico y pragmático. Se pensó en la emisora porque es un espacio fuera de las aulas y de los procesos académicos regulares, que dinamiza el aprendizaje y fortalece las competencias lectoras y escritoras (CLE) de las estudiantes de la institución. Se diseñó un curso en Moodle, como herramienta que desarrolla y fortalece las habilidades propias de las competencias lectoras y escritoras de las estudiantes que participan del proyecto, al tiempo que la emisora se mantiene en funcionamiento en el colegio.

Palabras clave: emisora escolar, lectura y escritura, habilidad comunicativa, sistematización de experiencias, radio.

\begin{abstract}
This research, developed at the Master in Education of the Antonio Nariño University (UAN), in Bogotá-Colombia, developed a strategy that mediated in the development of communicative reading and writing skills in the students of the Liceo Femenino Mercedes Nariño IED (LFMN-IED), a public school. The research sought to expose the process that students developed with the communication skills of reading and writing in the dynamics of the school radio station; in addition, it sought to establish, through an evaluative instrument, the initial and achieved skills reading and writing at the semantic, syntactic and pragmatic levels. The school radio station was thought as a space outside the classrooms and regular academic processes, which would boost learning and strengthen the reading and writing skills of the students. A Moodle course was designed, with the "pretext" of working in the processes of the school radio station, developing and strengthening the reading and writing skills of the students who participated in the project, while the radio station was working at school.
\end{abstract}

Keywords: school radio station, reading and writing, communication skills, systematization of experiences, radio.

\title{
Introducción
}

El Liceo Femenino Mercedes Nariño IED, institución oficial ubicada al sur de Bogotá (Colombia), con una población aproximada de 1200 estudiantes para el año 2018, en el nivel de básica primaria, tiene diferencias en sus niveles socioeconómicos y culturales.

Los resultados históricos del colegio en las pruebas Saber en el área de lenguaje durante los años 2013 a 2016 presentaron diferencias estadísticas significativas reflejadas en una disminución constante de los promedios. Haciendo una lectura de estos resultados, se pudo concluir que los procesos lectoescriturales de las niñas de grado tercero y quinto presentaban deficiencias, que a través de los años se acentuaban. Estas conclusiones son evidenciadas también a través de la experiencia que tiene el investigador en las prácticas pedagógicas cotidianas con las estudiantes en el colegio.

La posibilidad de interactuar con un proyecto pedagógico que se desarrollaba en la institución en medios de comunicación escolar permitió, entonces, desarrollar una propuesta de investigación para integrar dichos medios,

Revista PAPELES • ISSN 0123-0670 • Vol. 12(23) • pp. 98-111 • Enero-junio de 2020 
específicamente, la emisora en el desarrollo de las habilidades comunicativas de las niñas del liceo, por medio de la pregunta problema: ¿cómo contribuir al desarrollo de competencias comunicativas de escritura y lectura mediante el trabajo con la emisora escolar en estudiantes de básica primaria del Liceo Femenino Mercedes Nariño?

\section{Marco teórico}



La "columna vertebral" de esta investigación se plantea en tres aspectos:

Primero: el relacionado con las experiencias afines, organizadas en tres grupos: uno, analizó los medios de comunicación como una estrategia implementada en los procesos de formación en algunos lugares de Europa, especialmente en España. Destacan propuestas como las de Fernández Cárdenas (2011), Melgarejo Moreno y Rodríguez Rosell (2013) y Contreras Pulido et al. (2016). Un segundo grupo de investigaciones se enfoca en América Latina y el Caribe e involucra los medios de comunicación, como es el caso de las propuestas adelantadas por Cicilia Krohling-Peruzzo (2006), Quintanilla Espinoza y Ferreira Cabrera (2010), Peñarrieta Bedoya (2010), Verdugo
Rosero (2013), Surin (2014) y Cormenzana et al. (2015). Un tercer grupo muestra los aportes colombianos a la investigación sobre la relación de los medios de comunicación y la academia; entre ellos resaltan los resultados encontrados por Monsalve Upegui et al. (2011), Mosquera Ibarguen (2013), Valderrama Pulido y Reyes Bayona (2016), Palacios Ballen (2012), Poveda y Santiago Tibavizco (2015) y Romero Moreno (2015), que sirvieron como referente para esta investigación.

Segundo: el que confrontó los resultados en pruebas nacionales e internacionales en competencias comunicativas de lectura y escritura (CLE). En Colombia, el Icfes ${ }^{1}$ realiza las pruebas $\mathrm{Saber}^{2}$, en las que, de acuerdo con sus informes, los resultados arrojados en lenguaje en tercero y quinto del Liceo Femenino Mercedes Nariño IED (LFMN-IED) muestran que en el promedio en los últimos 5 años, únicamente el $11 \%$ ha logrado alcanzar el nivel avanzado (ver figura 1).

Por otro lado, revisando los resultados Colombia en las pruebas PISA $^{3}$, que evalúa las competencias de los estudiantes en tres áreas del conocimiento: lectura, matemáticas y ciencias naturales a nivel internacional, se

1 Instituto colombiano para el fomento de la educación superior.

2 Se aplican en los grados tercero, quinto, noveno y undécimo y buscan, según el MEN, ayudar al mejoramiento de la calidad de la educación en Colombia mediante la aplicación de evaluaciones periódicas.

3 Programa para la Evaluación Internacional de Alumnos de la OCDE (PISA, por sus siglas en inglés: Programme for International Student Assessment).

Universidad Antonio Nariño • Facultad de Ciencias de la Educación 
Figura 1. Resultados históricos de las Pruebas Saber tercero y quinto del Liceo Femenino Mercedes Nariño
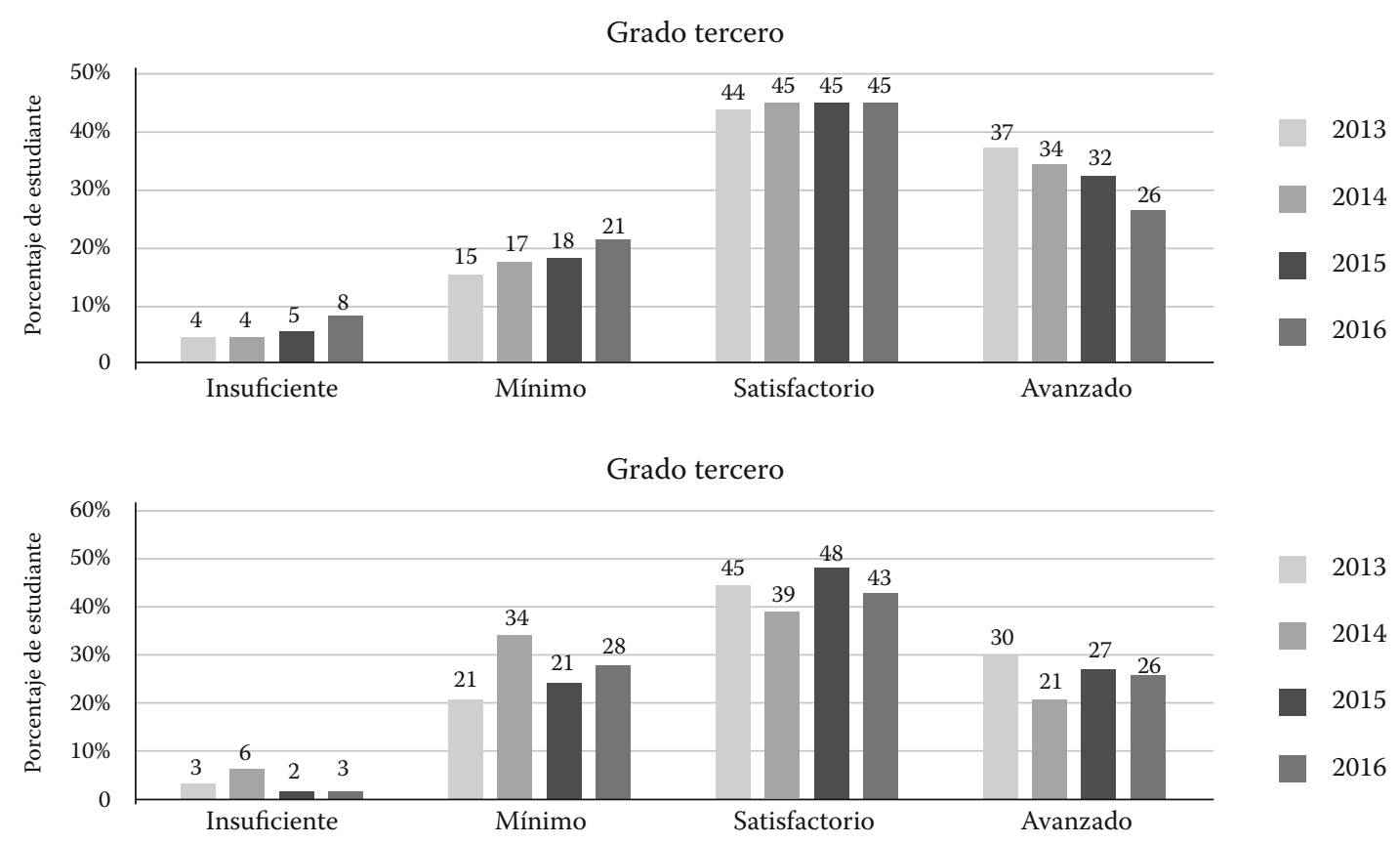

Fuente: elaboración propia

Figura 2. Resultados históricos de lectura (Pruebas PISA)

Resultados históricos en Lectura Pruebas PISA

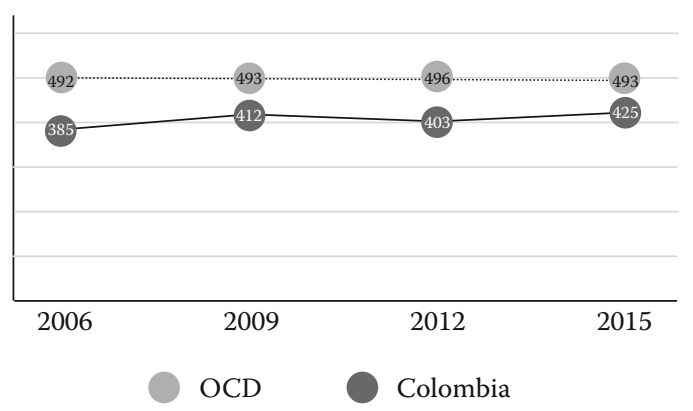

Fuente: elaboración propia

encontró, tal como lo muestra la figura 2, que el país siempre ha tenido bajos resultados en lectura con respecto al promedio de la OCDE.

Este análisis identificó fortalezas y habilidades de los estudiantes durante su periodo escolar. Las pruebas mostraron que no es cuánto sabe un estudiante en matemáticas o lenguaje sino cómo aplica los conocimientos que tiene de estas áreas en la vida real, qué tan competentes son para la vida, qué habilidades posee y cómo las usaría en el mundo actual.

Tercero: en el último aspecto se hizo un contraste de diferentes orientaciones legales para estos dos procesos (lectura y escritura), entre las que se podrían mencionar las establecidas en la Ley General de Educación, los Lineamientos y Estándares Curriculares, el Plan Nacional de Lectura y Escritura, algunas recomendaciones sobre el uso de las TIC en la escuela y los compromisos adquiridos en la Declaración de Incheon (2017).

Todas estas políticas públicas dejan claro el papel que tienen las habilidades comunicativas en el ámbito escolar, pero también en otros espacios fuera de la academia; y al mismo tiempo muestran las directrices adoptadas en el país para fortalecerlas y convertirlas en un bastión del proceso educativo y social. 
Figura 3. Diseño metodológico de la estrategia

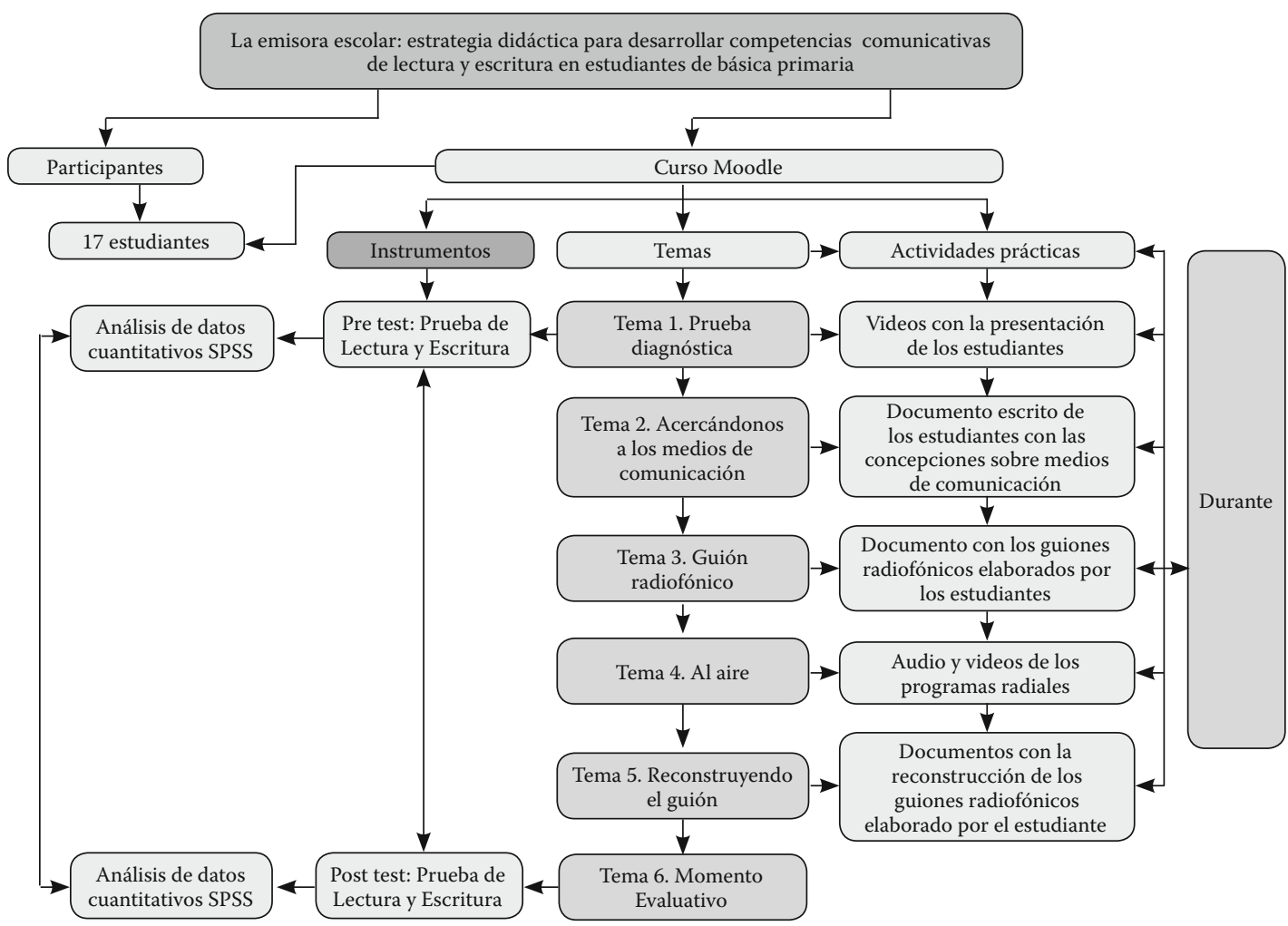

Fuente: elaboración propia

\section{Diseño metodológico}

El proceso metodológico se describe en la figura 3, en la que se ve organizado el curso de Moodle, los instrumentos de recolección y el método de análisis de la información obtenida.

Esto busca cumplir con los objetivos propuestos al diseñar una estrategia didáctica para convertir la emisora escolar en un espacio que desarrolle de las HCLE de los estudiantes que participan del proyecto de prensa escolar en el LFMN-IED, analizar los resultados de las pruebas aplicadas (pre-post) en las CLE a las estudiantes que participan del proyecto, analizar y comparar los resultados de los participantes de la estrategia en estas competencias de acuerdo con los niveles establecidos dentro del instrumento (sintáctico, semántico y pragmático) y evaluar la estrategia mediante un ejercicio de sistematización de experiencias con los datos recogidos en el proceso.

\section{Recolección de datos}

Para la recolección de datos se hizo necesario optar por diferentes instrumentos que permitan obtener la información necesaria y pertinente para la investigación. Desde esta perspectiva, se diseñó una prueba pretest y postest y se procedió a sistematizar la experiencia desde un enfoque anecdótico y cronológico (en la medida de las posibilidades).

En lo concerniente a la prueba (pre y postest), se hizo un pilotaje en la forma de la misma con estudiantes de la institución con características similares a quienes participarían de la misma. Para ello, se realizó la selección de 30 preguntas de las pruebas Saber de tercero y quinto de 2015 diseñadas por el ICFES (disponibles en línea), que indagan por las competencias semánticas, sintácticas y pragmáticas de las estudiantes en los niveles mínimo, satisfactorio y avanzado. 
Este instrumento fue aplicado en línea a todas las niñas que participaban del proyecto, al inicio y al final de la intervención, y se evalúo (ver tabla 1) con una puntuación de acuerdo con el nivel de complejidad de la pregunta.

Tabla 1. Matriz de criterios de evaluación para la prueba pretest y postest

\begin{tabular}{|l|l|c|c|c|}
\hline Grado & $\begin{array}{c}\text { Competencia/ } \\
\text { Nivel }\end{array}$ & Semántica & Sintáctica & Pragmática \\
\hline \multirow{3}{*}{$3^{\circ}$} & Mínimo & 1 & 2 & 3 \\
\cline { 2 - 5 } & Satisfactorio & 2 & 4 & 6 \\
\cline { 2 - 5 } & Avanzado & 3 & 6 & 9 \\
\hline \multirow{2}{*}{$5^{\circ}$} & Mínimo & 2 & 4 & 6 \\
\cline { 2 - 5 } & Satisfactorio & 4 & 8 & 12 \\
\cline { 2 - 5 } & Avanzado & 6 & 12 & 18 \\
\hline
\end{tabular}

Fuente: elaboración propia

La sistematización de la experiencia, por otra parte, ofreció la posibilidad de realizar una exploración a una propuesta pedagógica, la cual, desde el interior de la práctica, permitió reflexionar desde un quehacer pedagógico y de una vivencia al interior de la institución, en la que se destacó la relevancia de la gran variedad de estrategias empleadas en el proceso de formación integral en las niñas.

\section{Diseño del curso en Moodle}

El curso se desarrolló de manera virtual, por medio de la plataforma Moodle, a la cual fueron matriculadas todas las estudiantes participantes del proyecto, con previa autorización de los acudientes. De esta forma, los materiales a trabajar estuvieron disponibles en Moodle para todas las participantes. También se dio una charla introductoria para el acceso a la plataforma y al curso propiamente dicho. El autor diseñó el curso, compuesto por seis temas, de los cuales cada uno contiene los siguientes elementos: nombre del tema, objetivo, participantes y organización, duración (tiempo para desarrollar el tema), desarrollo del tema, actividades prácticas, instrumentos utilizados y análisis de datos (forma en que se van a usar).

\section{Resultados}

Se presentan los resultados obtenidos a la luz de la metodología propuesta en dos partes: la primera es la sistematización de la experiencia y la segunda son los aportes del instrumento pretest y postest a la estrategia de la emisora escolar.

\section{Sistematización de la experiencia}

La sistematización se enfocó en ordenar el conjunto de objetivos educativos, metas, recursos, tiempos, proyección y evaluación implementada. Esto apuntó a realizar un proceso de reflexión crítica y óptima del proyecto de prensa escolar "Centro de Noticias Liceístas" y desde allí construir un nuevo conocimiento, teniendo como punto de partida la práctica.

Sistematizar esta experiencia implicó organización y ordenamiento de la información, permitiendo la construcción de conocimiento a partir de la descripción de los procesos y las actividades llevadas a cabo en la institución.

Se estableció, entonces, que este proceso se realizaría de manera anecdótica y en la medida de las posibilidades de forma cronológica, lo cual permitiría, posteriormente, extraer unas conclusiones del trabajo realizado y plantear posibles ajustes para continuar con el proyecto, expandirlo a otros espacios institucionales o replicarlo en instituciones educativas diferentes.

Para ayudar con esto, el proceso se organizó en tres años: 2016 al 2018. En cada uno de ellos se presentaron las situaciones más relevantes para la investigación y para el proyecto pedagógico institucional.

Cuando se procedía a iniciar el análisis de los datos cualitativos que permitirían evaluar la estrategia, por medio de triangulación y 
análisis documental, se presentó la pérdida de una clave de almacenamiento de datos, por lo que solo se pudo acceder a la información almacenada en la nube. Se estableció que lo anterior se podría solucionar con un análisis robusto de los resultados. Se procedió, entonces, a realizarlo desde un ejercicio de estadística descriptiva que mostraría los aportes de la estrategia al desarrollo de CLE, como se podrá ver en el siguiente apartado.

\section{Aportes del instrumento (prueba pretest y postest) a la estrategia}

A continuación, se presentan los resultados obtenidos con la aplicación de la prueba aplicada en pre y post (prueba Saber de tercero y quinto del año 2015). Para ello, es necesario explicar brevemente cómo se interpretó este instrumento.
En la tabla 2 se identifican 12 tipos de preguntas (primera columna izquierda), luego en la columna 2 la cantidad total de preguntas que corresponden a la primera columna. Las columnas 3 , 4 y 5 muestran la cantidad de preguntas por nivel correspondientes a cada competencia.

Con respecto a los resultados arrojados por el pretest, se obtuvieron los puntajes absolutos de la tabla 3 para las 17 participantes del proyecto, y se registró como tendencia una mediana de 97 puntos $(\mathbb{\nabla}=97)$, una media de 99,82 puntos $(\mathbb{Q}=99,82)$; además de ser unos resultados amodales. Se puede observar que el valor mínimo corresponde a Q5 con 69 puntos y el mayor puntaje a C2 con 162 puntos.

De la tabla 2 se realiza la campana de Gauss de la figura 4, que muestra la distribución normal de los puntajes resultantes de evaluar la prueba.

Tabla 2. Matriz de la estructura de la prueba por competencias

\begin{tabular}{|c|c|c|c|c|}
\hline \multirow{2}{*}{ Tipo de pregunta } & \multirow{2}{*}{ Cantidad total } & \multicolumn{3}{|c|}{ Pregunta por nivel } \\
\hline & & Min. & Ava. & Satis. \\
\hline Lec. Semántica $3^{\circ}$ & 5 & 2 & 1 & 2 \\
\hline Lec. Sintáctica $3^{\circ}$ & 1 & & & 1 \\
\hline Lec. Pragmática $3^{\circ}$ & 2 & & & 2 \\
\hline Lec. Semántica $5^{\circ}$ & 3 & & 1 & 2 \\
\hline Lec. Sintáctica $5^{\circ}$ & 4 & & 1 & 3 \\
\hline Lec. Pragmática $5^{\circ}$ & 1 & & & 1 \\
\hline Escr. Semántica $3^{\circ}$ & 6 & & 2 & 4 \\
\hline Escr. Sintáctica $3^{\circ}$ & 0 & & & \\
\hline Escr. Pragmática $3^{\circ}$ & 1 & & & 1 \\
\hline Escr. Semántica $5^{\circ}$ & 1 & & 1 & \\
\hline Escr. Sintáctica $5^{\circ}$ & 3 & & 2 & 1 \\
\hline Escr. Pragmática $5^{\circ}$ & 3 & & 2 & 1 \\
\hline Total & 30 & 2 & 10 & 18 \\
\hline
\end{tabular}

Fuente: elaboración propia

Tabla 3. Resultados de la prueba pretest

\begin{tabular}{|c|c|c|c|c|c|c|c|c|c|c|c|c|r|r|r|r|r|r|r|r|r|r|r|}
\hline Seudonimo/participante & C1 & C2 & C3 & C4 & Q1 & Q2 & Q3 & Q4 & Q5 & Q6 & Q7 & Q8 & Q9 & Q10 & Q11 & Q12 & Q13 & X & Y \\
\hline Puntaje absoluto pre test. & 82 & 162 & 112 & 97 & 108 & 75 & 105 & 95 & 69 & 113 & 114 & 81 & 78 & 85 & 116 & 132 & 73 & 97 & 99,8 \\
\hline
\end{tabular}

Fuente: elaboración propia 
Figura 4. Distribución de los puntajes del pretest

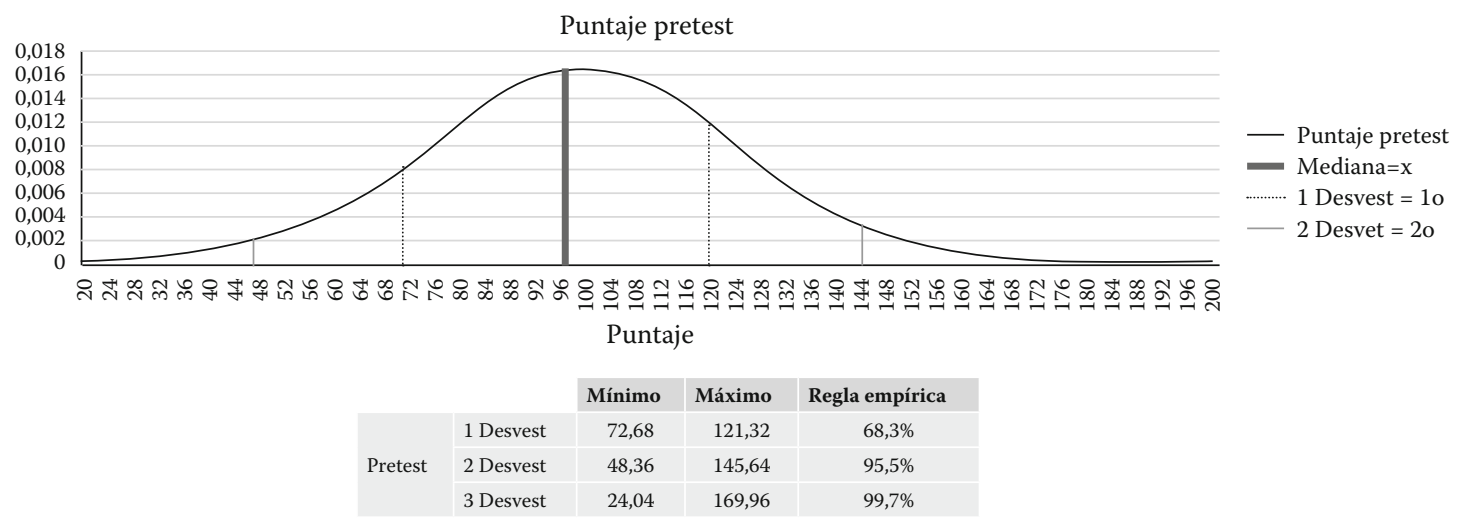

Fuente: elaboración propia

Según la regla empírica, el 68,3\% de los datos se ubican a $1 \nabla$ por encima y por debajo de la medida de tendencia central $(\mathbb{\Xi})$, para este caso $\mathbb{Q}=97 / 100$ y la $\mathbb{Q}=24,32$. Asumiendo que los datos estén distribuidos normalmente, es perceptible que un estudiante normal obtenga un puntaje entre 72 y 121 , es decir que su nivel de competencia sea satisfactorio; lo que deja un $15,9 \%$ restante con un puntaje inferior a 72 puntos, como estudiantes con bajo rendimiento en los procesos evaluados (nivel mínimo), que contrasta con otro 15,9\% que obtiene puntajes superiores a 121 (nivel avanzado) y muestra altos estándares en los procesos lectoescriturales.

Estos resultados también son un punto de partida interesante para medir el impacto de la propuesta metodológica y el desarrollo de la misma, puesto que muestran que existe un déficit en las competencias de lectura y escritura, al apenas superar la $\nabla$ el promedio de respuestas acertadas, lo que valida la intervención a realizar.

Ya en relación con el análisis del postest, se muestran en la tabla 4 los puntajes absolutos obtenidos al responder la prueba postest. Se observa ahora que el valor mínimo alcanzado es de 41 puntos y el mayor de 171, lo que da una media $\square=112,59$ y una mediana $\square=116$; datos que son amodales.

Los valores medios se aproximan a la mediana. $\mathrm{Al}$ realizar las comparaciones entre los resultados de la tabla 3 (pretest) y la tabla 4 (postest) se observan comportamientos irregulares en los resultados presentados en la figura 5 , y el hecho más relevante es que la línea de tendencia del postest marca un ascenso a lo largo de las diferentes participantes de la prueba.

Figura 5. Comparación de los resultados obtenidos en el pre y postest

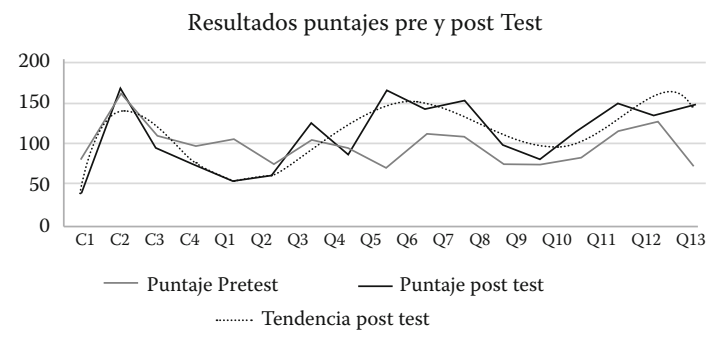

Fuente: elaboración propia

Tabla 4. Resultados de la prueba postest



Fuente: elaboración propia

Revista PAPELES • ISSN 0123-0670 • Vol. 12(23) • pp. 98-111 • Enero-junio de 2020 
Figura 6. Comparación de la distribución normal de los resultados obtenidos en el pre y postest

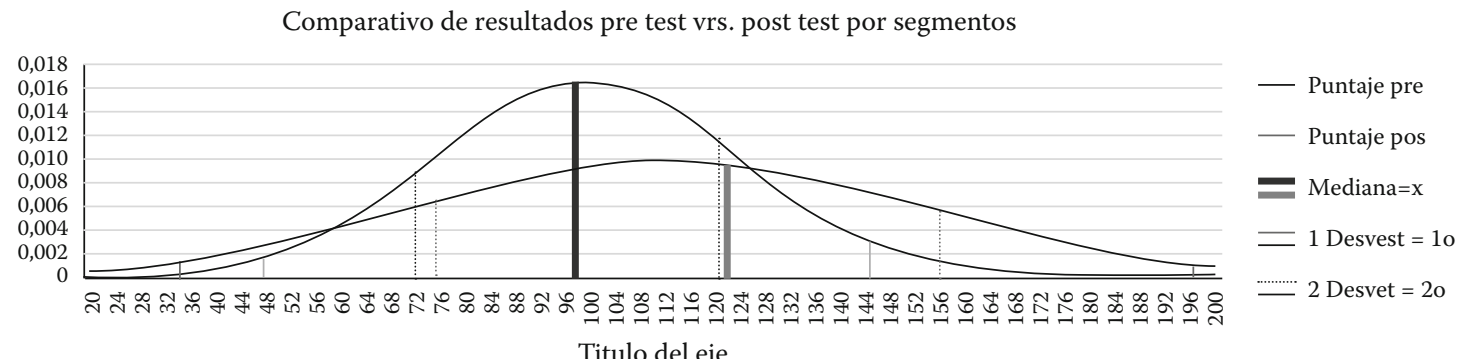

Titulo del eje

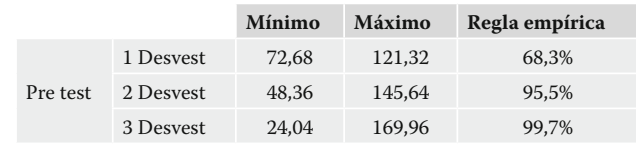

\begin{tabular}{|l|l|r|r|c|}
\hline \multirow{3}{*}{ Pre test } & & Mínimo & Máximo & Regla empírica \\
\hline \multirow{3}{*}{ 1 Desvest } & 75,25 & 156,75 & $68,3 \%$ \\
\hline & 2 Desvest & 34,49 & 197,51 & $95,5 \%$ \\
\hline \multirow{2}{*}{ 3 Desvest } & $-6,26$ & 238,26 & $99,7 \%$ \\
\hline
\end{tabular}

Fuente: elaboración propia

Con base en la información reflejada en figura 5 y en las tablas 3 y 4 , se realiza el comparativo de las distribuciones normales de las dos pruebas mediante la campana de Gauss (figura 6), la cual arrojó como resultado que la media $\otimes$ pasó de 99,82 a 112,59, lo que significó un avance de 12,76 puntos en promedio por estudiante. También se puede deducir que la mediana $\triangle$ creció un 20\% al pasar de 97 a 116 puntos.

Como se observa en la figura 6, el grupo que queda dentro de la $1 \nabla$ en el postest, aumentó su rango cerca del $67 \%$ con respecto al pretest; esto significa que el grupo de estudiantes con resultados satisfactorios son aquellos que obtuvieron entre 75 y 156 puntos, mientras que quienes están por encima de este valor se clasifican con niveles avanzados y dejan, así, a quienes obtengan menos de 75 puntos con un nivel de competencia mínimo.

Otro hecho relevante es que hasta el primer punto de inflexión (60 puntos) los resultados del postest están por encima del pretest. A partir de este punto, en la zona de promedio (nivel satisfactorio) disminuyeron los puntajes porque a partir del segundo punto de inflexión (126 puntos) mejoraron considerablemente por encima del pretest, posicionando a más estudiantes en el nivel avanzado. Finalmente, se observa cómo las medidas de tendencia central $(\nabla y \rrbracket)$ y las desviaciones estándar (a 1》

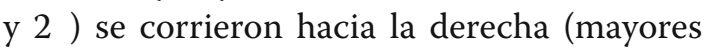
puntajes), lo que indica que la gran mayoría de las estudiantes mejoraron sus puntajes.

A partir de esta información, y con la intención de validarla, se construyó la matriz de la tabla 5 , que permite realizar la comparación del comportamiento de los resultados en deciles.

Si se lee esta matriz de manera vertical, se encontrará el porcentaje de estudiantes que se ubica en cada decil en la prueba del pretest; si, por el contrario, la lectura se realiza de manera horizontal, podrá identificarse el porcentaje de participantes que se ubicó en cada decil al finalizar el postest.

Se resaltaron en gris claro los puntos de intersección entre las dos pruebas, los cuales arrojaron que el 5,88\% de las estudiantes $(\mathrm{N}=1$ aprox.) se mantiene en el mismo decil. Por otro lado, se han marcado en gris oscuro los porcentajes que se ubicaron por debajo en los deciles con respecto a los obtenidos en el pretest, los cuales corresponden apenas al 35,29\% ( $\mathrm{N}=6$ estudiantes aprox.). Finalmente, se marcaron en gris medio aquellos porcentajes que se ubicaron en deciles mayores con respecto a la primera prueba, lo que mostró que un $58,82 \%$ de las estudiantes ( $\mathrm{N}=10$ aprox.) mejoraron sus resultados. Este análisis muestra nuevamente una tendencia al observar que, 
Tabla 5. Matriz de comparación del comportamiento de los resultados en deciles

\begin{tabular}{|c|c|c|c|c|c|c|c|c|c|c|}
\hline & & & & & & Pre & test & & & \\
\hline & Deciles & $60-70$ & $70-80$ & $80-90$ & $90-100$ & $100-110$ & $110-120$ & $130-140$ & $160-180$ & Total general \\
\hline & $40-50$ & $0,00 \%$ & & $5,88 \%$ & & & & & & $5,88 \%$ \\
\hline & $50-60$ & $0,00 \%$ & & & & $5,88 \%$ & & & & $5,88 \%$ \\
\hline & $60-70$ & $0,00 \%$ & $5,88 \%$ & & & & & & & $5,88 \%$ \\
\hline & $70-80$ & $0,00 \%$ & & & $5,88 \%$ & & & & & $5,88 \%$ \\
\hline & $80-90$ & $0,00 \%$ & $5,88 \%$ & & $5,88 \%$ & & & & & $11,76 \%$ \\
\hline$\ddot{\uplus}$ & $90-100$ & $0,00 \%$ & & $5,88 \%$ & & & $5,88 \%$ & & & $11,76 \%$ \\
\hline$\vec{\omega}$ & $110-120$ & $0,00 \%$ & & $5,88 \%$ & & & & & & $5,88 \%$ \\
\hline & $120-130$ & $0,00 \%$ & & & & $5,88 \%$ & & & & $5,88 \%$ \\
\hline & $130-140$ & $0,00 \%$ & & & & & & $5,88 \%$ & & $5,88 \%$ \\
\hline & $140-150$ & $0,00 \%$ & $5,88 \%$ & & & & $11,76 \%$ & & & $17,65 \%$ \\
\hline & $150-160$ & $0,00 \%$ & & & & & $5,88 \%$ & & & $5,88 \%$ \\
\hline & $160-170$ & $5,88 \%$ & & & & & & & & $5,88 \%$ \\
\hline & $170-180$ & $0,00 \%$ & & & & & & & $5,88 \%$ & $5,88 \%$ \\
\hline & Total general & $5,88 \%$ & $17,65 \%$ & $17,65 \%$ & $11,76 \%$ & $11,76 \%$ & $23,53 \%$ & $5,88 \%$ & $5,88 \%$ & $100,00 \%$ \\
\hline
\end{tabular}

Fuente: elaboración propia

en total, el 60,74\% de las estudiantes, es decir, $\mathrm{N}=11$ aprox., se mantuvieron o mejoraron sus resultados frente a los obtenidos en la primera prueba.

El análisis se hizo también desde las competencias lectoras y escritoras. Para entender el análisis de los resultados por competencias se proyectaron los puntajes correspondientes a los diferentes niveles de lectura (semántica, sintáctica y pragmática) y la media ( $)$ obtenida en las pruebas (pre y postest) para esas preguntas. Al revisar los resultados obtenidos en las pruebas, con respecto a la lectura, se puede evidenciar que una vez que las estudiantes han presentado las dos pruebas existe una mejoría en los procesos de lectura relacionados con el campo semántico, en el que el avance fue del 5,9\%; el campo sintáctico optimizó sus resultados en un $10,3 \%$.

Sin embargo, en el campo pragmático no hubo progreso. Esta comparación la podemos encontrar de manera más evidente en la figura 7. También podemos inferir que, aunque los porcentajes en el nivel semántico, y en algún momento en el nivel sintáctico, no son superiores al $50 \%$, la capacidad de entender el uso del lenguaje en diferentes situaciones a través de la lectura (nivel pragmático) sí es alto, por lo que se asume que el no saber qué dice el texto o cómo se organiza no es impedimento para que se pueda entender cuál es su propósito, y, así, establecer un efectivo acto comunicativo.

Figura 7. Comparación de resultados en la competencia escritora

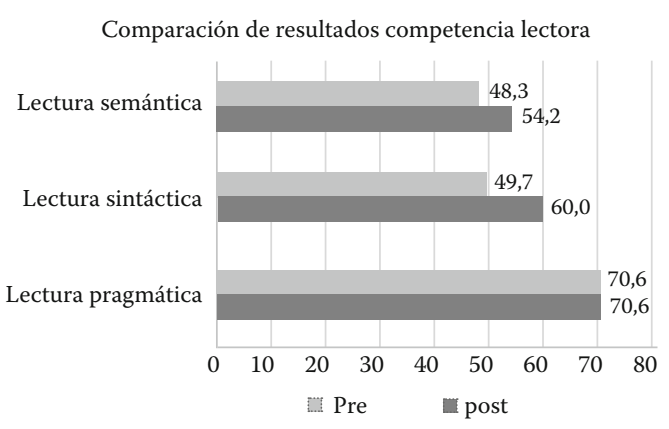

Fuente: elaboración propia 
Tal como se hizo para la competencia lectora, se realizó un análisis de los puntajes correspondientes a la competencia escritora, tanto para el total de la prueba en los niveles semántico,


de los resultados del pretest y del postest.

Aquí, a diferencia de la competencia lectora, existen diferentes comportamientos entre el pre y post frente a los niveles analizados, tal como se puede ver en la figura 8.

Figura 8. Comparación de resultados del pos-test vs. los resultados distritales

Comparación de resultados competencia escritora

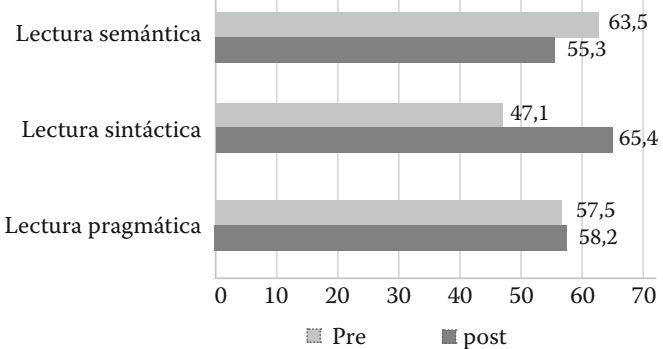

Fuente: elaboración propia

En primer lugar, en los diferentes niveles y en las distintas pruebas el porcentaje de respuestas correctas supera el 50\% (salvo en el pretest, referente al nivel sintáctico que solo alcanza el $47,1 \%)$, suponiendo que la habilidad escritora de las participantes en el proyecto es buena y alcanza los mínimos establecidos en los estándares de competencias.

Luego, podríamos ver que los resultados muestran una disminución de un $8,2 \%$ en lo que respecta al nivel semántico (caso atípico), mientras que en el nivel sintáctico existe un avance que alcanza el 18,3\%, mucho mayor al $0,7 \%$ que se registró en la competencia pragmática.

Finalmente, se observa un mayor desarrollo de los procesos escritores relacionados con el nivel sintáctico, reflejado en la capacidad de mejorar la escritura de las participantes desde la organización del texto.
Por su parte, el análisis comparativo del postest respecto a los resultados distritales y nacionales se realizó en términos porcentuales (figura 9).

Figura 9. Comparación de resultados del postest vs. los resultados nacionales



Fuente: elaboración propia

Como se observa en la figura 9, la tendencia de los resultados del postest se encuentra normalmente en el mismo rango de los resultados distritales y nacionales; más aún, en algunas preguntas por encima de estos, tal como es el caso de las preguntas 2, 3, 5, 6, 8, $9,12,24$ y 25 . Adicionalmente, existen preguntas en las que se percibe una diferencia amplia entre los resultados de las pruebas aplicadas y los porcentajes obtenidos en las pruebas distritales y nacionales. 


\section{Conclusiones}

Para presentar las conclusiones se tendrán en cuenta dos ejes: el primero, las que surgen de la sistematización de la experiencia, y el segundo, las inferencias a partir de la prueba a la estrategia. De esta forma, se da cuenta de la evolución de la propuesta pedagógica que duró 3 años.

Implementar un proyecto dentro de una institución educativa no es un proceso sencillo porque existen múltiples variables que el docente no puede controlar y que desestabilizan la puesta en marcha del mismo; más complejo aún, cuando de este se pretende realizar una investigación con fines académicos para la educación superior. Estos elementos proporcionan nuevos retos que ponen a prueba la capacidad de liderazgo, improvisación, flexibilidad y adaptación de quien se encuentra al frente del mismo. Al tratarse de una investigación con un análisis de datos de tipo descriptivo, quedan por fuera muchos elementos que se pudieron observar durante la implementación de la propuesta y que atañen directamente a una investigación cualitativa.

En la investigación se presentaron situaciones que dificultaron el desarrollo de la propuesta, como garantizar el acceso y la conexión a internet para todos los participantes, la disponibilidad de tiempo para la ejecución de los temas, que pudieron incidir en los resultados obtenidos, que no son variables analizables en esta investigación, pero sí mencionables desde la sistematización de la experiencia. Del ejercicio real se pudo concluir que las estudiantes sienten una gran motivación para participar en proyectos que les permitan mostrar su talento y trabajo a la comunidad liceísta; también se evidenció una preocupación constante de las participantes activas para cumplir con las tareas asignadas. La estrategia de la emisora escolar se puede convertir en una herramienta de trabajo pedagógico que potencie no solo las CLE, como lo proponía esta investigación, sino también otras: comunicativas (como la oralidad y la escucha), de interacción y responsabilidad social, etc.

A pesar de que los sujetos de esta investigación son "nativos digitales", es evidente una falta de conocimiento de procesos básicos mediados por las TIC, el uso del computador con fines académicos, el manejo de programas de paquete de oficina, elementos para la navegación en la red (carga y descarga de archivos) y un hábito de trabajo consciente y concentrado; estos fueron algunos otros aspectos que se observaron en la investigación. Se logró, entonces, desde la experiencia, y sin haberlo programado, resaltar lo más importante de este proceso, que es la historia que hay detrás de la investigación: la vida al interior del proyecto de prensa escolar Centro de Noticias Liceístas (CNL), y lo poderoso que puede volverse un ambiente virtual construido con bases sólidas y referentes teóricos que permiten cada día hacer ajustes y mejorar los contenidos para que cumplan satisfactoriamente el propósito con el que fueron diseñados.

Y el segundo: las inferencias que se pueden realizar desde los aportes hechos por la prueba a la estrategia. Como se pudo observar, la tendencia del postest, analizada con la función polinómica en grado sexto, permite inferir que existe un aumento cercano al 74\% (R2= 0,744). Estos resultados muestran avances en los procesos de las estudiantes que no se pueden asociar directamente con la estrategia al carecer de un grupo control o de un análisis documental cualitativo.

Los resultados obtenidos en el pretest y el postest, así como la ejecución de las temáticas propuestas en el curso, son susceptibles al nivel de formación académica (grado), edad y posibilidades económicas y sociales que tienen las diferentes participantes del proyecto. Se puede suponer que es por esta razón que el grupo correspondiente al grado quinto (estudiantes 
con seudónimo Q) obtuvo siempre mejores resultados al tener un nivel académico más alto que las estudiantes del grado cuarto (participantes con el seudónimo C); de igual modo, aspectos individuales afectaron el resultado final.

El diseñó de la prueba tipo Icfes, que se aplicó en dos momentos (pretest y postest), permitió que el análisis de los datos recogidos en las pruebas mostrara que, primero, el 64\% de los participantes en la propuesta de intervención obtuvieron un rendimiento mayor en el postest con respecto a sus propios resultados del pretest. Estos representan un incremento promedio del 39\% en respuestas acertadas.

Podríamos concluir que el avance en las capacidades relacionadas con los niveles semánticos y sintácticos les da a las estudiantes la capacidad de entender qué dice el texto y cómo se organiza, pero, sobre todo, las habilita para el uso correcto del lenguaje en diferentes situaciones a través de la lectura (nivel pragmático), entendiendo cuál es el propósito del texto y estableciendo actos comunicativos efectivos y eficaces.

\section{Recomendaciones y prospectiva de futuros estudios}

El desarrollo de las habilidades comunicativas siempre ha estado en el radar de los procesos pedagógicos dentro y fuera del aula, elemento que queda señalado en las directrices que se formulan en políticas públicas y en las prácticas de los docentes en los diferentes niveles y sectores de la educación. Ahora bien, encontrar nuevas formas de hacerlo se convierte en una fuente inagotable de trabajo investigativo. Descubrir cómo los medios de comunicación, enfocados en la escuela, proporcionan un campo amplio de trabajo, puesto que existen diferentes matices que se pueden imprimir a estos, es una tarea compleja que desde la investigación académica se puede abordar. Hablar, escuchar, leer y escribir son competencias que se pueden medir desde la mirada cuantitativa (en términos de avances, comparaciones y probabilidades), pero los son más aún desde la perspectiva cualitativa, que permitirá identificar, además de algunos aspectos mencionados anteriormente, el cómo aprenden, para qué y por qué lo hacen, así como presentar componentes claves dentro del proceso.

Por último, el ejercicio de la sistematización de experiencias permitirá que prácticas educativas exitosas se conviertan en el insumo para fortalecer el quehacer pedagógico de muchos docentes que buscan, en documentos como estos, herramientas que se puedan implementar en sus propias aulas y escuelas, para enriquecer los saberes propios y de los estudiantes de diferentes niveles. La yuxtaposición de saberes, prácticas, escenarios, poblaciones y metodologías engrandece la profesión docente y da un significado al ejercicio de la misma cuando se impacta de manera positiva la vida, y no solo escolar, de cada uno de los estudiantes a quienes permea.

\section{Referencias}

Contreras Pulido, P., Duarte Hueros, A. y Guzmán Franco, M. D. (2016). Propuestas educomunicativas desde Uniradio: de infantil a la Universidad. EDMETIC, 5(2), 182-201.

Goodman, K. (1986). El lenguaje Integral (3a. Ed). Buenos Aires: Aique Grupo Editor.
ICFES.(s/f).Resultados Saber $3^{\circ}, 5^{\circ}$ y $9^{\circ}$. Bogotá: ICFES. Recuperado de http://www2.ICFES interactivo.gov.co/ReportesSaber359/historico/reporteHistoricoComparativo.jspx

Llobera, M. (1995). Competencia comunicativa: documentos básicos en la enseñanza de lenguas extranjeras. Madrid: Edelsa. 
McLuham,M.(2018).Medios calientesy Medios Fríos. Ciudad de México: Sabersinfin. Recuperado de https://www.sabersinfin.com/ articulos/documentos/282-medios-calientes-y-medios-frs-en-marshall-mcluhan

Ministerio de Educación Nacional (MEN). (1994). Ley General de Educación 115 de 1994. Bogotá: MEN.

Ministerio de Educación Nacional (MEN). (1998a). Lineamientos curriculares. Bogotá: MEN.

Ministerio de Educación Nacional (MEN). (1998b). Lineamientos Curriculares para Lengua Castellana. Bogotá: MEN.

Ministerio de Educación Nacional (MEN). (2006). Estándares Básicos de Competencias en Lenguaje, Matemáticas, Ciencias y Ciudadanas. Bogotá: MEN.

Ministerio de Educación Nacional (MEN). (2011). Plan Nacional de Lectura y Escritura de Educación Inicial, Preescolar, Básica y Media. Bogotá: MEN.

Ministerio de Educación Nacional de Colombia (MEN). (2017). Pruebas Saber. Bogotá: MEN.
Organisation for Economic Co-operation and Development (OECD). (2018). Programme for International Student Assessment. París: OECD.

UNESCO (2015). Education 2030: Incheon Declaration and Framework for Action for the implementation of Sustainable Development Goal 4: Ensure inclusive and equitable quality education and promote lifelong learning opportunities for all. Incheon: Corea del Sur: UNESCO.

Valdés, H., Treviño, E., Acevedo, C. G., Castro, M., Carrillo, S., Costilla, R., ... Pardo, C. (2008). SERCE - Los aprendizajes de los estudiantes de América Latina y el Caribe. Resumen Ejecutivo del Primer Reporte de Resultados del Segundo Estudio Regional Comparativo y Explicativo. Bogotá: Salesianos Impresiones.

Valencia Cobos, J., Camargo Ariza, K., Cabrera Durán, Y., Beltrán Zabaleta, E., González, J., Barrios Oliveros, L., ... Badillo Peralta, A. (2013). Estrategias para el Fortalecimiento de las TIC en las Instituciones Educativas Oficiales de Colombia. Bogotá: Universidad del Norte-Observatorio de Educación del Caribe Colombiano. 